\title{
Hematuria Secondary to Benign Prostatic Hyperplasia: Retrospective Analysis of 166 Men Identified in a Single One Stop Hematuria Clinic
}

\author{
Nikhil Vasdev Ashish Kumar Rajan Veeratterapillay Andrew C. Thorpe \\ Department of Urology, Freeman Hospital, Newcastle upon Tyne, UK
}

\section{Key Words \\ Benign prostatic hyperplasia $\cdot$ Hematuria}

\begin{abstract}
Introduction: Hematuria secondary to benign prostatic hyperplasia (BPH) can occur due to a vascular primary gland itself or due to the vascular re-growth of the prostate following a transurethral resection of the prostate (TURP). We aim to evaluate the clinical presentation and management in patients within both these groups. Materials and Methods: We retrospectively archived the data of 166 men diagnosed with hematuria secondary to $\mathrm{BPH}$ from our hematuria clinic database from March 2003 and March 2006. The 166 patients were divided into 2 groups: Group I $(n=94)$ hematuria with no previous TURP; Group II $(n=72)$ hematuria with previous TURP. The clinical management in both groups included reassurance, commencement of a 5 -alpha reductase inhibitor (finasteride) or a primary TURP in Group I or re-do TURP in Group II. Results: The median age was 73 years (range 45-94 years) for both groups. Outcomes combined for both groups included: reassurance alone in $26 \%(n=43)$, finasteride in $51 \%(n=84)$ and TURP in $12 \%(n=19)$. Patients managed with reassurance alone or TURP had no further episodes of hematuria. At a mean follow-up was 18 months (range 7-22 months), 2 patients treated with finasteride re-bled but did require further intervention. A further 2 men elected to stop finasteride due to erectile dysfunction and gynecomastia
\end{abstract}

\section{KARGER}

Fax +41613061234

E-Mail karger@karger.ch

www.karger.com
(C) 2012 S. Karger AG, Basel

1015-9770/12/0063-0146\$26.00/0

Accessible online at:

www.karger.com/cur respectively. Conclusion: BPH can present with hematuria. Following re-evaluation in a hematuria clinic, the lack of any subsequent cancer diagnosis in these patients suggests that repeat hematuria investigations should be carefully re-considered.

Copyright $\odot 2012$ S. Karger AG, Basel

\section{Introduction}

Hematuria is a common clinical presentation with a community prevalence of 1 in 40 patients [1] and is estimated to account for 4-20\% of all urological hospital visits [2]. The gradual rise in patient referrals to urological clinics is attributed to the increased use of urine dipstick analysis in the community [3]. Current recommendations from the Department of Health, United Kingdom presume that occult pathology may be present following hematuria presentation and as such should be investigated [4]. Further studies advocate urgent investigations of hematuria via a 'fast-track one-stop hematuria clinic' [4].

Common causes of hematuria in men include urinary infection, urological malignancy and benign prostatic hyperplasia (BPH). The latter diagnosis often results in vas- 
cular enlargement of the prostate leading to hematuria. In clinical practice, these patients present at a designated hematuria clinic resulting in extensive investigations. Variations exist within the literature as to the proportion of patients with normal investigations. Khadra et al. [5] quoted a no diagnosis rate of $56.6 \%$ in males while Edwards et al. [3] suggested a higher rate of $76.7 \%$.

Treatment for BPH involves medical or surgical management options. A transurethral resection of the prostate (TURP) is the one of the most common types of surgical management offered. However, despite this intervention patients can re-present with further hematuria following a TURP. Often, this results in further clinical assessment at the hematuria clinic, which escalates the economic burden and patient anxiety following hematuria investigation. Murakami et al. [6] suggested that if patients with persistent hematuria are followed then a significant proportion will be shown to have undiscovered neoplasia. However, Khadra et al. [5] suggested that if the initial investigation was thorough then the chance of missing significant pathology is small. Furthermore, some studies have suggested that prolonged bleeding can result in hematuria in otherwise normal patients [7, 8]. Others have suggested anti-coagulant therapy may result in hematuria in as many as $40 \%$ of cases [8].

We therefore aim to compare diagnostic and treatment outcomes in patients with BPH. We aim to specifically compare patients who have received a TURP in the past with those presenting for the first time. We hypothesize that BPH patients - re-presenting with hematuria following a TURP - do not warrant further investigation as this rarely provides any new pathology resulting in further treatment.

\section{Patients and Methods}

We retrospectively analyzed data from 1,730 patients who attended a 'one-stop' hematuria clinic at the Freeman Hospital, Newcastle-upon-Tyne between April 2003 and March 2006. From 1,730 patients, 1,061 patients were male $(61.3 \%)$ and were recognised as having hematuria secondary to $\mathrm{BPH}$ and no other pathology defined. Patients were referred to our clinic through the urgent 'two-week' hematuria referral form available to all general practitioners and via an out-patient referral process available to all departments within the scope of Newcastle and surrounding areas. This out-patient referral process was not necessarily conducted within 2 weeks. The inclusion criteria were the presence of visible and invisible hematuria in a male patient referred to our hematuria clinic.

Evaluation consisted of the recording of basic demographics, history, examination, blood tests, urinalysis, urine culture and cytology. In addition patients underwent a renal ultrasound, either

Hematuria Secondary to Benign Prostatic Hyperplasia
Table 1. Hematuria presentations

\begin{tabular}{lcc}
\hline & Group I & Group II \\
\hline Painless hematuria & $57(60.6 \%)$ & $58(80.6 \%)$ \\
Painful hematuria & $6(6.4 \%)$ & $5(6.9 \%)$ \\
Clot retention & $1(1.1 \%)$ & - \\
Asymptomatic microscopic & $13(13.8 \%)$ & $1(1.4 \%)$ \\
Symptomatic microscopic & $16(17.0 \%)$ & $7(9.7 \%)$ \\
Gross per urethral bleed & $1(1.1 \%)$ & $1(1.4 \%)$ \\
\hline
\end{tabular}

an intravenous urogram or kidney, ureter, and bladder X-ray and flexible cystoscopy.

Patients were subdivided into 2 groups based on final coded diagnosis. Group I contained 94 patients with BPH only and no previous TURP. Group II contained 72 patients with BPH only and a previous TURP.

\section{Results}

\section{Basic Demographics and Referrals}

Within Group I (BPH and no previous TURP) the mean age of presentation was 71 years (range 45-94 years). This was comparable to the group with previous TURP where the mean age was 75 years (range 56-92 years). The degrees of hematuria were identified by type of hematuria, the associated pain and any additional clinical features. These are summarised in table 1 below. Patients referred with painless hematuria formed the largest subgroup within both cohorts. The vast majority (98.9\%) had no prior urological diagnoses with 1 patient identified as having a solitary renal cyst.

Referrals were categorised based on the 'two-week cancer referral' process. Differences between the 2 groups were identified. In Group I, patients diagnosed with vascular prostates and no previous TURP, 55.3\% (52/94) of patients were referred urgently for suspected cancer. A significant increase was noted with Group II where patients had already had a TURP $(54 / 72,75.0 \%)$.

\section{Symptoms and Initial Referral Diagnosis}

The majority of patients in both groups presented with signs and symptoms of lower urinary tract symptomology (LUTS). This was comparable where Group I showed $80.9 \%$ identifying LUTS as their primary symptom and Group II where $86.1 \%$ of patients identified LUTS as the primary presentation. Further diagnoses included urinary infections and calculi. All patients in both cohorts were 
diagnosed with vascular BPH with 1 patient in Group I diagnosed with bladder calculi.

\section{Investigations}

Initial investigations included prostate specific antigen (PSA), serum full blood count and urea and electrolytes and urinalysis. PSA analysis was performed in selected patients in both groups. The proportion of patients receiving a PSA was identified as $30.9 \%$ in Group I and $15.3 \%$ in Group II. Differences were noted between the 2 cohorts in the PSA values obtained. In Group I the mean PSA was $5.4 \mathrm{ng} / \mathrm{ml}$ (IQR: 0.9-7.4); in Group II the mean PSA was $4.7 \mathrm{ng} / \mathrm{ml}$ (IQR: 4.4-13.7). The proportion of normal renal function was comparable in both cohorts: 94.6\% (Group I) and 95.8\% (Group II).

Following urinalysis, all urine samples were sent to microscopy, culture and cytological analysis. The negative urine culture rate was also comparable between the 2 cohorts. In patients with no previous TURP, $91.7 \%$ of patients had negative urine microscopy and $91.5 \%$ negative urine cultures. This compared with the second cohort where $94.7 \%$ of patients had negative urine microscopy and $88.9 \%$ had negative urine cultures.

No cytological abnormalities were detected in $89.4 \%$ of samples in Group I and $86.1 \%$ of samples in Group II. However, after factoring inadequate and unavailable samples the rate of suspicious cytology showed differences between the 2 cohorts. In Group I, 3.2\% of samples were suspicious; a significantly greater proportion of $9.7 \%$ were identified in Group II.

Further assessment at the hematuria clinic involved a renal ultrasound scan, an intravenous urogram and a flexible cystoscopy (table 1). Again, the results were comparable between the 2 groups of patients in all 3 investigative modalities. There were a greater proportion of normal renal ultrasounds and flexible cystoscopies in Group I.

\section{Diagnosis and Further Management}

In both cohorts there was no cancer diagnosis. The final diagnosis was hematuria secondary to BPH in Group I and hematuria secondary to vascular re-growth of the prostate following a previous TURP in the other group. The mean follow-up was 18 months (range 7-22 months) in both subgroups patients were managed either with reassurance alone $-37 \%(\mathrm{n}=63)$, with finasteride $-51 \%$ $(\mathrm{n}=84)$ or TURP $-11 \%(\mathrm{n}=19)$. Two patients treated with finasteride re-bled but required no intervention. A further 2 men elected to stop finasteride due to erectile dysfunction and gynecomastia respectively (table 2).
Table 2. Management outcomes following investigation

\begin{tabular}{lcc}
\hline & Group I & Group II \\
\hline Discharged with no further follow-up & $27(28.7 \%)$ & $15(20.8 \%)$ \\
Finasteride & $43(45.7 \%)$ & $41(56.9 \%)$ \\
$\alpha$-blocker & $5(5.3 \%)$ & - \\
TURP & $9(9.6 \%)$ & - \\
Re-do TURP & - & $10(13.9 \%)$ \\
Further out-patient review & $5(5.3 \%)$ & $5(6.9 \%)$ \\
Died during investigation period & $3(3.2 \%)$ & - \\
Unknown/not documented & $2(2.1 \%)$ & $1(1.4 \%)$ \\
\hline
\end{tabular}

\section{Discussion}

Hematuria remains a common presentation in male patients of varying ages and is estimated to account for 4-20\% of all urological hospital visits [2]. Causes of hematuria in men include urinary infection, urological malignancy and BPH. Vascular enlargement of the prostate often leads to hematuria in male patients. In clinical practice, these patients present at the hematuria clinic and following investigations, if a diagnosis of benign prostatic enlargement is noted, then treatment in the form of medical or surgical options is offered.

Our aim was to identify any differences in clinical assessment outcomes between male patients, presenting with hematuria, pre- and post-TURP. We hypothesized that BPH patients re-presenting with hematuria following a TURP - do not warrant further investigation as this rarely provides any new pathology resulting in further treatment.

Our findings show the 2 subgroups were comparable clinically. Painless macroscopic hematuria was the most common presentation to our hematuria clinic which is in keeping with current accepted documented findings. The majority of investigations were comparable between the groups; however, more abnormal findings were noted post-TURP. The mean PSA was higher post-TURP, perhaps reflecting increased vascular re-growth of the prostate. However, renal function, urine microscopy, urine culture and urine cytology were all largely negative in both groups. Furthermore, imaging and cystoscopic findings were significantly unremarkable and subgroup analysis identified Group I patients, with no previous TURP, as having less significant identifiable pathology.

There were more patients post-TURP (75.0 vs. 55.3\%) referred under the urgent 'two-week' rule, reflecting the 
concerns of primary care physicians that hematuria may represent malignancy. However, the final diagnosis in both subgroups identifies no cancer diagnosis, suggesting overuse of the urgent 'two-week' referral pathway. It can be difficult, however, to identify potential malignancy in the primary care setting. Thus, urologists may have to explore better ways of identifying and assessing malignant causes of hematuria with further education of primary care physicians.

The final diagnosis was hematuria secondary to BPH in Group I and hematuria secondary to vascular regrowth of the prostate following a previous TURP in the other group. Certain trends can be identified from subsequent management. Conservative treatment options included reassurance and 5- $\alpha$ reductase inhibitors such as finasteride. Surgical options for persistent hematuria with LUTS were managed by TURP, which is the most common type of procedure offered. In this study $89 \%$ of patients required either reassurance or medical therapy in the form of finasteride, a 5- $\alpha$ reductase inhibitor. Only $11 \%$ required a TURP.

This cohort of 166 patients, both pre- and post-TURP, show investigations do not increase cancer diagnosis.
Furthermore, these results show that re-investigating hematuria does not offer any further information. However, there is obvious selection bias and this remains a small comparative population. Clearly, a larger, randomisedcontrolled trial would be ideal, but may present ethical dilemmas. Certain trends can be deduced and we propose that considering invasive and more expensive investigations should only be undertaken if the clinical picture appears suspicious.

\section{Conclusion}

BPH can represent with further hematuria. Conservative treatment options include reassurance and 5- $\alpha$ reductase inhibitors such as finasteride. Surgical options for persistent hematuria with LUTS can be managed by TURP. The lack of any cancer diagnoses in this cohort of patients suggests that repeat hematuria investigations of flexible cystoscopy and imaging are not necessarily required. We propose that invasive and more expensive investigations should only be undertaken if the clinical picture appears suspicious.

\section{References}

1 Ritchie CD, Bevan EA, Collier SJ: Importance of occult haematuria found at screening. Br Med J (Clin Res Ed) 1986;292:681-683.

-2 Mariani AJ, Mariani MC, Macchioni C, Stams UK, Hariharan A, Moriera A: The significance of adult hematuria: 1,000 hematuria evaluations including a risk-benefit and costeffectiveness analysis. J Urol 1989;141:350355.

-3 Edwards TJ, Dickinson AJ, Natale S, Gosling J, McGrath JS: A prospective analysis of the diagnostic yield resulting from the attendance of 4020 patients at a protocol-driven haematuria clinic. BJU Int 2006;97:301-305.
4 Guidelines for urgent referral of patients with suspected cancers. London, Department of Health, 2004.

5 Khadra MH, Pickard RS, Charlton M, Powell PH, Neal DE: A prospective analysis of 1,930 patients with hematuria to evaluate current diagnostic practice. J Urol 2000;163:524-527.

-6 Murakami S, Igarashi T, Hara S, Shimazaki J: Strategies for asymptomatic microscopic hematuria: a prospective study of 1034 patients. J Urol 1990;144:99-101.

-7 Kraus SE, Siroky MB, Babyan RK, Krane RJ: Hematuria and the use of non-steroidal antiinflammatory drugs. J Urol 1984;132:288290.
8 Benton O, Lazarchick J, Orak JK, Turner WR: Use of a bleeding time determination in the evaluation of unexplained hematuria. J Urol 1987;137:527-528.

$\checkmark 9$ Golin AL, Howard RS: Asymptomatic microscopic hematuria. J Urol 1980;124:389-391.

10 Rodgers $\mathrm{M}$, Nixon J, Hempel S, Aho T, Kelly J, Neal D, Duffy S, Ritchie G, Kleijnen J, Westwood M: Diagnostic tests and algorithms used in the investigation of haematuria: systematic reviews and economic evaluation. Health Technol Assess 2006;10: iii-iv, xi-259. 